\title{
Сравнение адсорбционных свойств мезопористых кремнеземов, допированных диспрозием, модифицированных медью и серебром, методом обращенной газовой хроматографии
}

\author{
(C) 2020 Филиппова Е.О., Шафигулин Р.В., \\ Виноградов К.Ю., Буланова А.В.
}

Самарский национальный исследовательский университет им. академика С.П. Королева, Самара

Поступила в редакцию 16.10.2020 г.

DOI: $10.17308 /$ sorpchrom.2020.20/3137

Темплатный синтез является перспективным методом получения мезопористых кремнеземов, позволяющий контролировать диаметр пор и площадь поверхности МСМ, благодаря значительной площади поверхности (700-1500 м²/г). Варьировать избирательность поглощения веществ возможно за счет жесткой структуры неорганической матрицы, доступности пор для проникновения объемных молекул органических веществ, а также возможность модифицирования материалов. Работы последних лет посвящены исследованиям в области получения новых мезопористых материалов, допированных редкоземельными элементами, материалов с особыми, малоизученными свойствами. Ранее авторами статьи были изучены адсорбционные свойства мезопористого силикагеля, допированного диспрозием и модифицированного никелем и установлено, что встраивание диспрозия в структуру материала изменяет адсорбционные свойства кремнезема.

Целью работы являлось сравнение адсорбционных свойств синтезированных мезопористых силикагелей, допированных диспрозием и модифицированных медью и серебром.

Темплатным методом синтезированы два образца мезопористого кремнезема: мезопористый кремнезем, допированный диспрозием и модифицированный медью $(\mathrm{Dy}-\mathrm{Cu} / \mathrm{MC})$ и мезопористый кремнезем, допированный диспрозием и модифицированный серебром (Dy-Ag/MC).

Текстурные и морфологические свойства образцов исследованы методами низкотемпературной адсорбции-десорбции азота, сканирующей электронной микроскопии, рентгенофазового анализа, масс-спектрометрии с индуктивно связанной плазмой и рентгенофлуоресцентного анализа. Установлено, что введение диспрозия в структуру кремнеземного мезопористого материала приводит к уменьшению площади его поверхности с 600 до $514 \mathrm{~m}^{2} /$ г. Синтезированные образцы характеризуются средним диаметром пор 3.4-7.2 нм. Синтезированный образец Dy- $\mathrm{Ag} / \mathrm{MC}$ характеризуется наибольшим удельным объемом пор $2.3 \mathrm{~cm}^{3} / \Gamma$.

Методом обращенной газовой хроматографии получены термодинамические характеристики адсорбции (дифференциальная теплота адсорбции и изменение стандартной дифференциальной молярной энтропии при адсорбции) для тестовых органических соединений. Установлено, что допирование и последующее модифицирование приводит к изменениям теплот адсорбции для соединений склонных к различным типам специфических взаимодействий. Сравнивая адсорбционные свойства синтезированных образцов, можно сделать вывод, что Dy-Cu/MC проявляет наиболее ярко выраженные адсорбционные свойства по отношению к исследованным полярным тестовым соединениям. На образце $\mathrm{Dy}-\mathrm{Cu} / \mathrm{MC}$ метанол адсорбируется с большей теплотой, чем этанол, что, вероятно, обусловливается низким значением объема пор образца.

Ключевые слова: мезопористые силикагели, допирование диспрозием, модифицирование медью и серебром, термодинамика адсорбции, обращенная газовая хроматография 


\section{Введение}

Темплатный синтез является перспективным методом получения мезопористых кремнеземов, позволяющий контролировать диаметр пор и площадь поверхности мезопористых материалов типа МСМ-41. Мезопористым силикагелям присущи большая площадь поверхности (700-1500 м²/г) и упорядоченная структура мезопор, благодаря вышеперечисленным характеристикам эти материалы характеризуются высокой адсорбционной способностью и могут являться перспективными адсорбентами. Варьировать избирательность поглощения веществ возможно за счет жесткой структуры неорганической матрицы, доступности пор для адсорбатов, а также возможности модифицирования материалов [1-6].

Работы последних лет посвящены исследованиям в области получения новых мезопористых материалов, допированных редкоземельными элементами, материалов с особыми, малоизученными свойствами [7-10]. Модификация поверхности мезопористых кремнеземов наночастицами переходных металлов значительно повышает их селективность и, в частности, они могут быть использованы как адсорбенты для очистки от токсичных веществ [11], для адсорбции различных биологически активных веществ, например, витаминов [12] и аминокислот [13-14]. Кроме того, модифицированные мезопористые силикагели могут использоваться в качестве резервуаров для хранения различных веществ [15-17].

Ранее авторами статьи [18] были изучены адсорбционные свойства мезопористого силикагеля, допированного диспрозием и модифицированного никелем, и установлено, что встраивание диспрозия в структуру материала изменяет адсорбционные свойства кремнезема.

Целью работы являлось изучение и сравнение адсорбционных свойств синтезированных мезопористых силикагелей, допированных диспрозием и модифицированных медью и серебром.

\section{Эксперимент}

Темплатным методом синтезированы два образца мезопористого кремнезема: мезопористый кремнезем, допированный диспрозием и модифицированный медью (Dy-Cu/MC) и мезопористый кремнезем, допированный диспрозием и модифицированный серебром (Dy-Ag/MC). Золь-гель синтез мезопористого силикагеля, допированного диспрозием (Dy/MC), проводили по методике, описанной в работе [19]. При модифицировании кремнезема, допированного диспрозием (Dy/MC) переходными металлами, образцы пропитывали спиртовым раствором сульфата меди и водным раствором нитрата серебра при перемешивании на магнитной мешалке. После этого образец, пропитанный сульфатом меди, нагревали в токе водорода в муфельной печи при $250^{\circ} \mathrm{C}$ в течение 4 часов до восстановления металла. Образец, пропитанный нитратом серебра, подвергали термической обработке в муфельной печи в воздушной атмосфере при $550^{\circ} \mathrm{C}$ в течение 4 часов до полного разложения нитрата серебра.

Форму и размеры полученных частиц мезопористых силикагелей исследовали методом сканирующей электронной микроскопии (СЭМ, марки электронного микроскопа CarlZeiss EVO 50, энергодисперсионная приставка X-Max 80). С помощью рентгенофлуоресцентного анализа (РФА) определяли наличие металлов в образцах катализаторов; методом спектрометрии с индуктивно-связанной плазмой (ICP) определяли концентрацию меди, серебра и диспрозия в структурах $\mathrm{Dy}-\mathrm{Cu} / \mathrm{MC}$ и Dy$\mathrm{Ag} / \mathrm{MC}$. В дополнение, наличие металлов в образцах подтверждалось методом рентгенофазового анализа (XRD). Были определены размеры пор и удельная площадь 
поверхности с использованием адсорбционного порозиметра Quantochrome Autosorb-1, удельную поверхность рассчитывали по модели Брунауэра-ЭмметаТэллера (БЭТ). Общий объем пор и распределение мезо- и макропор по размерам рассчитывали по десорбционной кривой с использованием модели БарретаДжойнера-Халенды (ВЈH).

Адсорбционные свойства полученных материалов изучали методом обращенной газовой хроматографии на хроматографе TraceGC с пламенно-ионизационным детектором; газ-носитель - гелий. Синтезированные образцы массой 0.19-0.2 г помещали в насадочную колонку длиной 50.2 см и внутренним диаметром 2 мм. В качестве адсорбатов использовали гексан, гептан, октан, бензол, циклогексен, метанол, этанол, нитрометан, ацетон, диэтиловый эфир, ацетонитрил. Исследования проводили в интервале температур 423-443 К. Первичные характеристики удерживания получали из 5 параллельных экспериментов с ошибкой не более $3 \%$. Для расчета чистого (эффективного) объема удерживания и константы адсорбционного равновесия использовали формулы, приведенные в работе [20].

\section{Обсуждение результатов}

1. Результаты физико-химических методов анализа синтезированных образцов. Полученные изотермы адсорбции и десорбции (IV тип) и распределение пор по размеру (модель ВЈН) представлены на рис. 1.

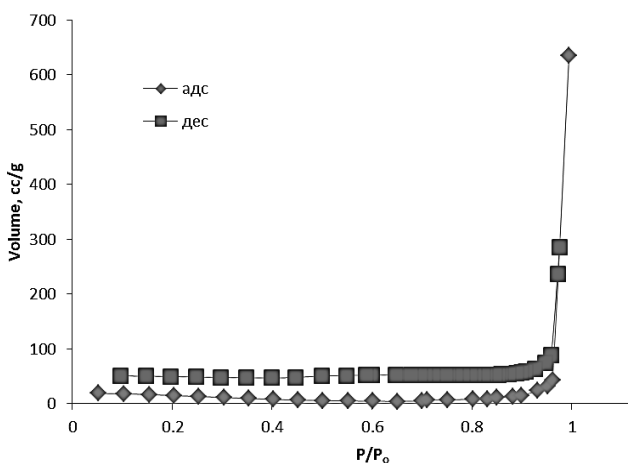

$\mathrm{a}$

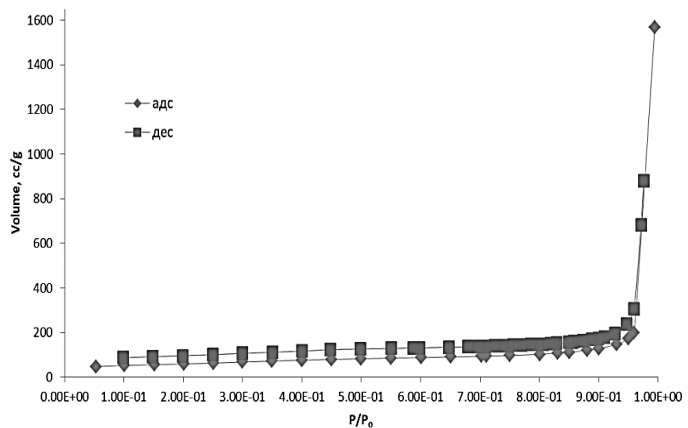

B

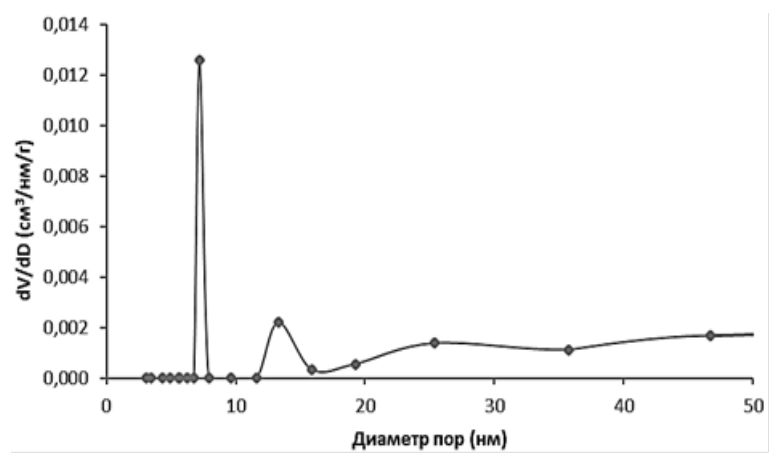

б

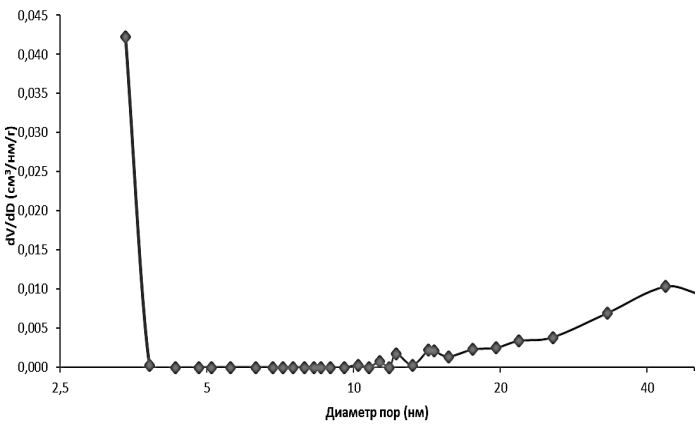

$\Gamma$

Рис. 1. Изотермы адсорбции-десорбции азота при $77 \mathrm{~K}$ и распределение пор по размерам (ВЈH-метод) для Dy-Cu/MC(1a, 1б), Dy-Ag/MC(1в, 1г).

Fig. 1. Nitrogen adsorption-desorption isotherms at $77 \mathrm{~K}$ and pore size distribution (BJH method) for Dy-Cu/MS (1a, 1b), Dy-Ag/ MS (1c, 1d)

B таблице 1 представлены текстурные характеристики полученных Dy$\mathrm{Cu} / \mathrm{MC}$ и $\mathrm{Dy}-\mathrm{Ag} / \mathrm{MC}$. 
Таблица 1. Значения удельной площади поверхности и текстурных характеристик пор для $\mathrm{MC}, \mathrm{Dy} / \mathrm{MC}, \mathrm{Dy}-\mathrm{Cu} / \mathrm{MC}, \mathrm{Dy}-\mathrm{Ag} / \mathrm{MC}$

Table 1. Specific surface area and texture characteristics of pores for MC, Dy/MC, Dy$\mathrm{Cu} / \mathrm{MC}, \mathrm{Dy}-\mathrm{Ag} / \mathrm{MC}$

\begin{tabular}{|c|c|c|c|}
\hline Название & $\mathrm{S}(\mathrm{BET}), \mathrm{M}^{2} / \Gamma$ & $\mathrm{V}_{\text {пор }}(\mathrm{BJH}$ des $), \mathrm{cm}^{3} / \Gamma$ & $\mathrm{D}_{\text {эф }}(\mathrm{BJH}$ des$), \mathrm{nm}$ \\
\hline $\mathrm{MC}$ & $600 \pm 30$ & 0.828 & $<4$ \\
\hline $\mathrm{Dy} / \mathrm{MC}$ & $514 \pm 25$ & 0.457 & 3.8 \\
\hline $\mathrm{Dy}-\mathrm{Cu} / \mathrm{MC}$ & $33 \pm 10$ & 0.998 & 7.2 \\
\hline $\mathrm{Dy}-\mathrm{Ag} / \mathrm{MC}$ & $208 \pm 12$ & 2.3 & 3.4 \\
\hline
\end{tabular}

Установлено, что введение диспрозия в структуру кремнеземного мезопористого материала приводит к уменьшению площади его поверхности с 600 до $514 \mathrm{~m}^{2} / \Gamma$. Синтезированные образцы характеризуются средним диаметром пор 3.4-7.2 нм. Образец Dy-Ag/MC характеризуется наибольшим удельным объемом пор $2.3 \mathrm{~cm}^{3} / г$. При модифицировании образца Dу/МС металлами резко падает удельная поверхность и увеличивается средний объем пор, по-видимому, снижение удельной поверхности синтезированных материалов обусловлено тем, что металлы преимущественно находятся в порах мезопористой матрицы.

Наличие диспрозия, меди и серебра и их концентрация в структурах Dy$\mathrm{Cu} / \mathrm{MC}$ и Dy-Ag/MC определены РФА- и ICP - методами (рис. 2).

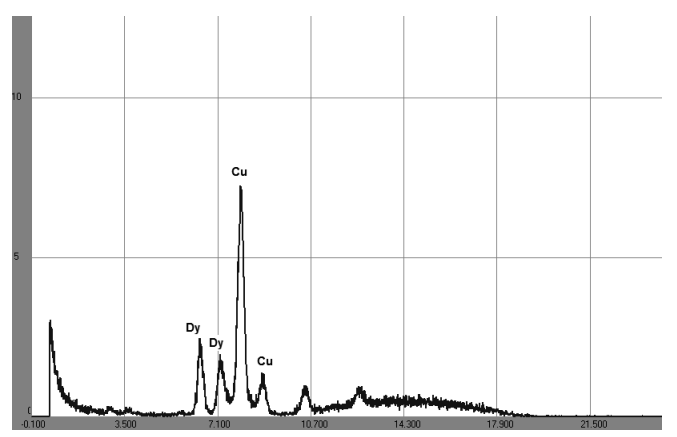

a

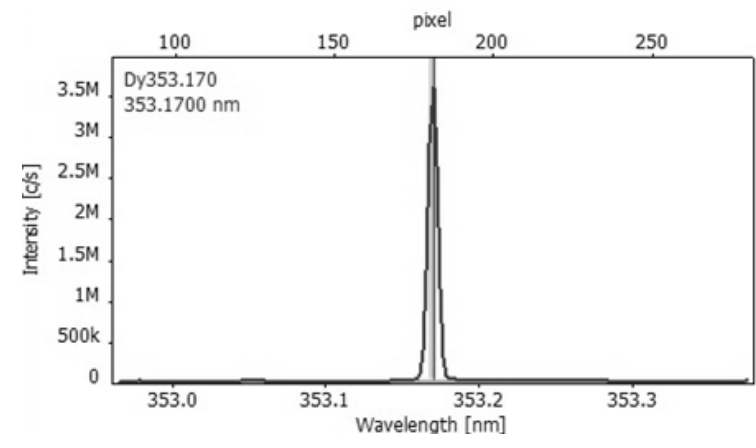

B

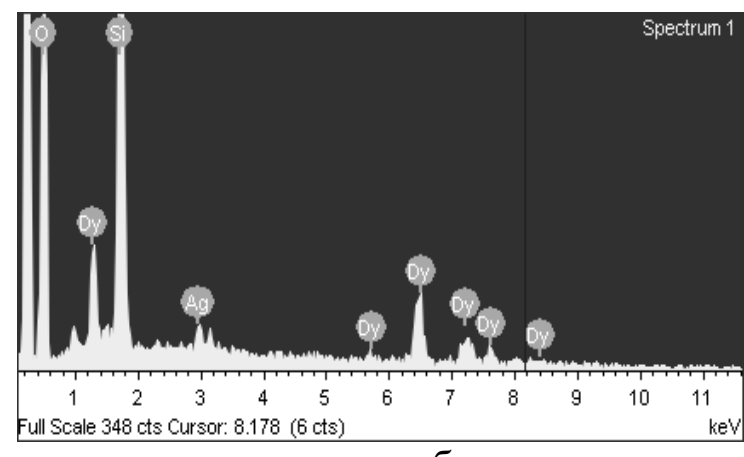

6

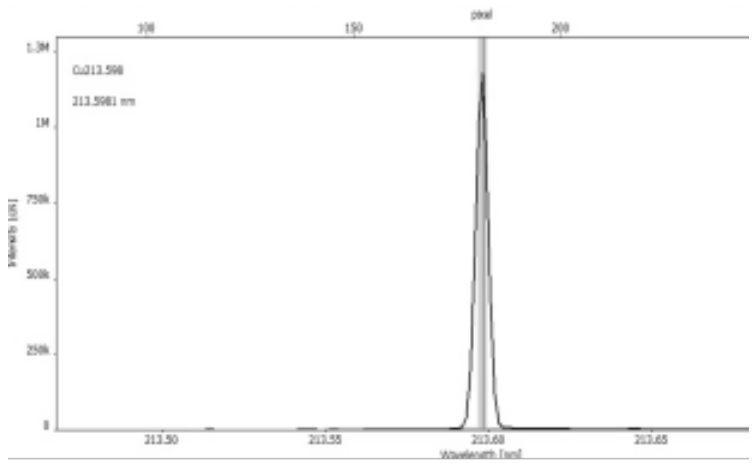

$\Gamma$

Рис. 2. РФА (a, б)- и ICP (в, г) спектры для образцов Dy-Cu/MC и Dy-Ag/MC.

Fig. 2. XRD (a, b) and ICP (c, d) spectra for Dy-Cu/MS and Dy-Ag/MS samples.

Концентрации Dy, Cu и Ag составили соответственно 1.3, 7 и 8\% по массе соответственно. Для образца Dy-Ag/MC дополнительно были проведены исследования с помощью рентгенофазового анализа (XRD) (рис. 3). 
Наличие в малоугловой области дифрактограммы характеристического пика свидетельствует об упорядоченной системе мезопор в структуре синтезированных образцов, соответствующей фазе типа МСМ-41. Присутствие серебра в форме металлической фазы с гранецентрированной металлической решеткой подтверждается наличием на дифрактограмме образца Dy-Ag/MC характерного рефлекса $\left(2 \Theta=39^{\circ}\right)$.

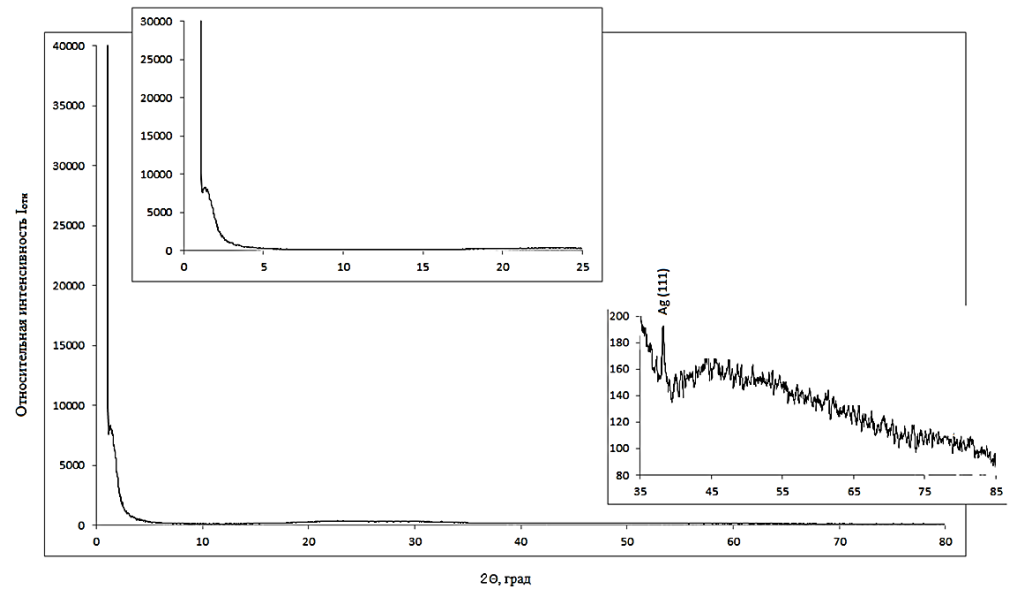

Рис. 3. Дифрактограмма для Dy-Ag/MC.

Fig. 3. Diffractogram for Dy-Ag/MS.

Фотографии синтезированных образцов, полученные методом СЭМ, представлены на рис.4.

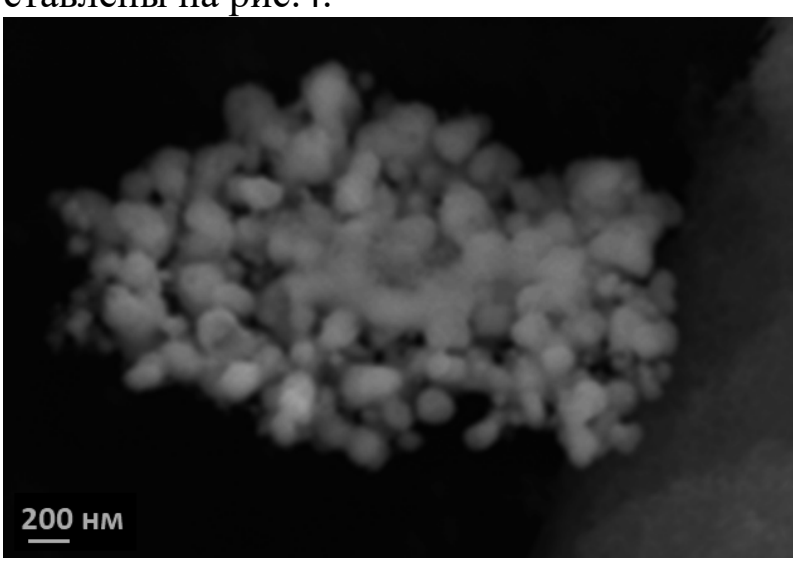

a

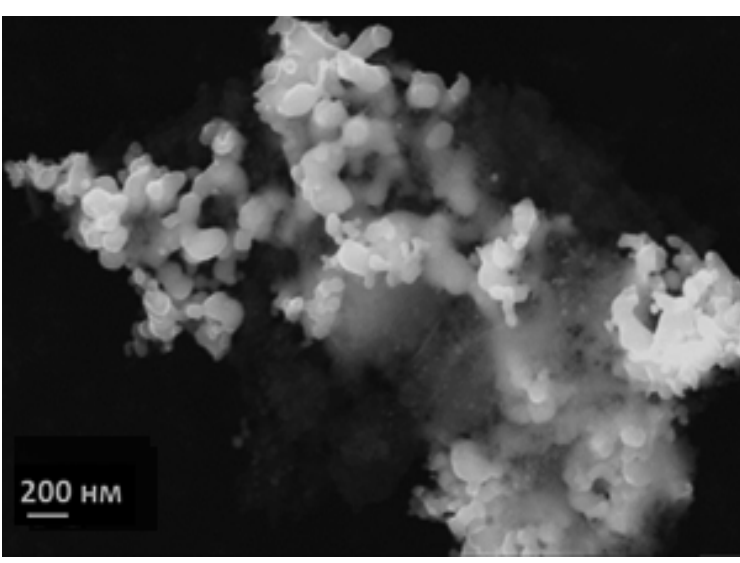

б

Рис. 4. СЭМ фотографии: $\mathrm{a}$ - MC, допированный диспрозием и модифицированный медью (Dy-Cu/MC); б - MC, допированный диспрозием и модифицированный серебром (Dy-Ag/MC).

Fig. 4. SEM photographs: a - MS doped with dysprosium and modified with copper (Dy-Cu/MS); b - MS doped with dysprosium and modified with silver (Dy-Ag/MS).

Установлено, что частицы имеют сферическую форму и их средний размер для образцов $\mathrm{Dy}-\mathrm{Cu} / \mathrm{MC}$ и $\mathrm{Dy}-\mathrm{Ag} / \mathrm{MC}$ составляет 230 и 100 нм, соответственно.

2. Изучение адсорбционных свойств Dy-Cu/MC и Dy-Ag/MC методом обращенной газовой хроматографии. Адсорбционные характеристики исследованных образцов характеризировали по теплотам адсорбции тестовых адсорбатов, которые рассчитывали, используя зависимость констант Генри сорбционного равновесия от температуры (рис. 5). 

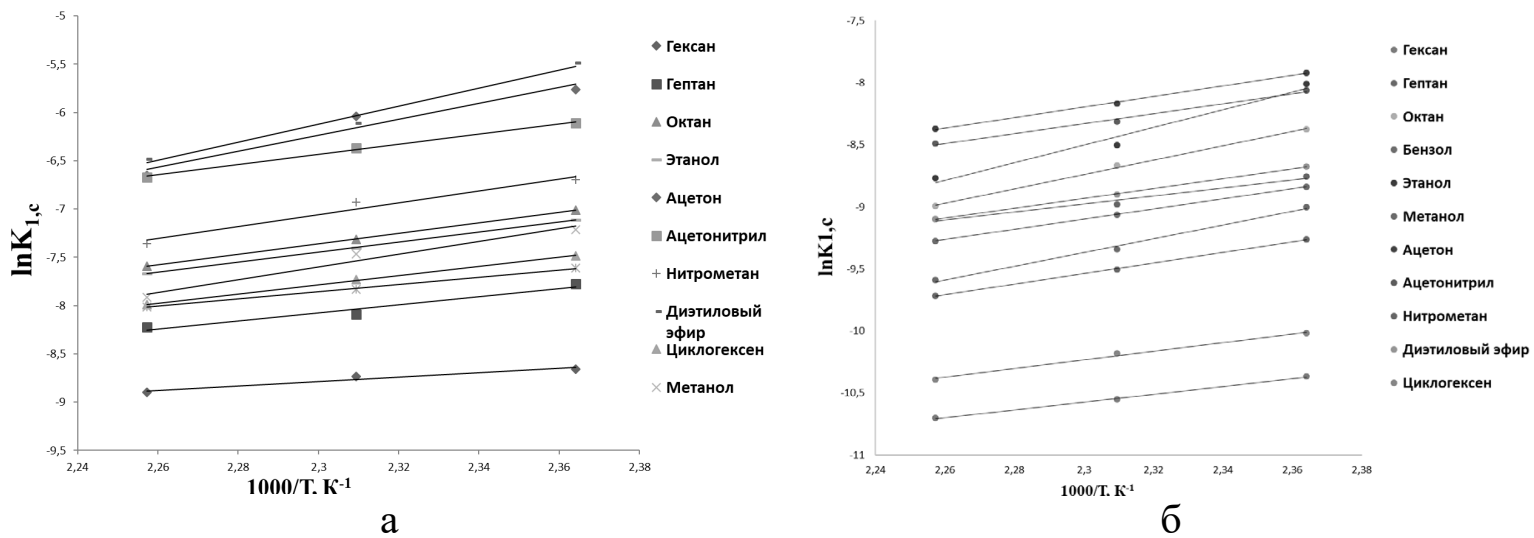

Рис. 5. Температурная зависимость логарифма константы Генри для тестовых органических соединений: а) Dy-Cu/MC, б) Dy-Ag/MC.

Fig. 5. Temperature dependence of the logarithm of Henry constant for tested organic compounds: a) Dy-Cu/MS, b) Dy-Ag/MS.

Температурные зависимости линейные в используемом диапазоне температур характеризуются высокими коэффициентами детерминации (не менее 0.988). В табл. 2 представлены значения теплот адсорбции $\left(\bar{Q}_{1}\right)$ и изменения дифференциальных энтропий адсорбции $\left(\Delta \bar{S}_{1}{ }^{9}\right.$ исследуемых соединений.

Таблица 2. Величины $\bar{Q}_{1}$ (кДж/моль), $\Delta \overline{\mathrm{S}}_{1}{ }^{0}($ Дж/(моль $\left.\cdot К)\right)$ для исследуемых адсорбатов на $\mathrm{MC}, \mathrm{Dy}-\mathrm{Ag} / \mathrm{MC}, \mathrm{Dy}-\mathrm{Cu} / \mathrm{MC}$

Table 2. $\overline{\mathrm{Q}}_{1}(\mathrm{~kJ} / \mathrm{mol}), \Delta \overline{\mathrm{S}}_{1}{ }^{\circ}\left(\mathrm{J} /\left(\mathrm{mol}^{*} \mathrm{~K}\right)\right)$ values for the studied adsorbates on MC, Dy$\mathrm{Ag} / \mathrm{MC}, \mathrm{Dy}-\mathrm{Cu} / \mathrm{MC}$

\begin{tabular}{|c|c|c|c|c|c|c|}
\hline \multirow{2}{*}{ Сорбат } & \multicolumn{2}{|c|}{$\mathrm{MC}$} & \multicolumn{2}{c|}{ Dy-Ag/MC } & \multicolumn{2}{c|}{ Dy-Cu/MC } \\
\cline { 2 - 7 } & $\overline{\mathrm{Q}}_{1}$ & $-\Delta \overline{\mathrm{S}}_{1}{ }^{0}$ & $\overline{\mathrm{Q}}_{1}$ & $-\Delta \overline{\mathrm{S}}_{1}{ }^{0}$ & $\overline{\mathrm{Q}}_{1}$ & $-\Delta \overline{\mathrm{S}}_{1}{ }^{0}$ \\
\hline Гексан & 30.1 & 113.6 & 26.0 & 147.7 & 18.7 & 99.6 \\
\hline Гептан & 33.2 & 114.4 & 35.5 & 160.9 & 35.1 & 131.4 \\
\hline Октан & 35.8 & 114.9 & 48.1 & 183.3 & 45.3 & 148.9 \\
\hline Метанол & 40.7 & 128.9 & 45.9 & 183.6 & 54.7 & 172.5 \\
\hline Этанол & 31.8 & 108.6 & 59.0 & 206.4 & 43.8 & 146.3 \\
\hline Бензол & 33.8 & 113.0 & 33.9 & 153.7 & 31.2 & 120.7 \\
\hline Нитрометан & 36.5 & 113.1 & 26.9 & 136.7 & 51.3 & 160.3 \\
\hline Ацетон & 64.2 & 164.0 & 35.1 & 149.0 & 69.0 & 193.9 \\
\hline Диэтиловый эфир & 56.6 & 150.5 & 32.8 & 104.3 & 77.5 & 204.3 \\
\hline Ацетонитрил & 49.3 & 136.9 & 33.2 & 145.7 & 43.9 & 138.1 \\
\hline Циклогексен & 33.8 & 114.5 & 29.0 & 151.7 & 39.8 & 139.7 \\
\hline
\end{tabular}

Из данных, приведенных в табл. 2 следует, что модифицирование металлами достаточно сильно изменяет адсорбционные свойства материалов. На рис. 6-7 приведены сравнительные диаграммы для теплот адсорбции исследуемых адсорбатов на синтезированных материалах.

Наблюдается резкий рост теплот от гексана к октану на модифицированных металлами материалах (более 10 кДж/моль). По-видимому, введение металлов усиливает дисперсионные взаимодействия неполярных линейных углеводородов с поверхностью адсорбента. Адсорбция бензола и циклогексена на исследованных адсорбентах характеризуется практически одинаковыми теплотами адсорбции; некоторое увеличение теплоты наблюдается на $\mathrm{Dy}-\mathrm{Cu} / \mathrm{MC}$ для циклогексена. 


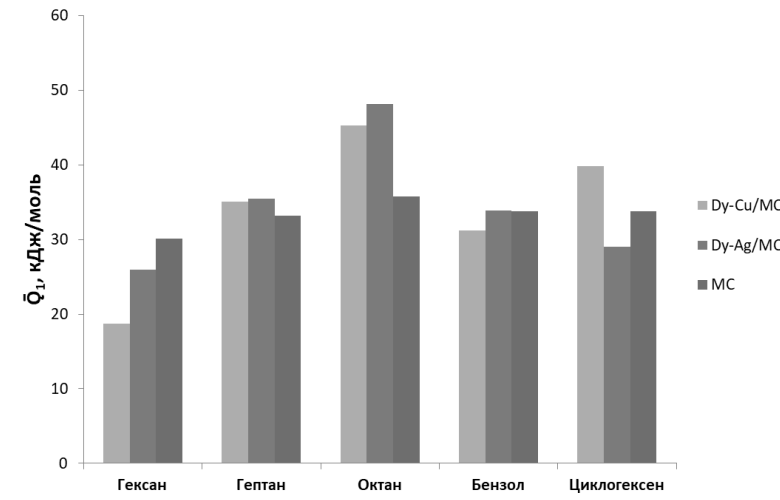

Рис. 6. Влияние модификатора на величины теплот $\left(\overline{\mathrm{Q}}_{1}\right)$ адсорбции углеводородов на адсорбентах MC, Dy-Ag/MC и $\mathrm{Dy}-\mathrm{Cu} / \mathrm{MC}$.

Fig. 6. Influence of the modifier on the heat of adsorption $\left(\bar{Q}_{1}\right)$ of hydrocarbons on MS adsorbents, Dy-Ag/MS and $\mathrm{Dy}-\mathrm{Cu} / \mathrm{MS}$.

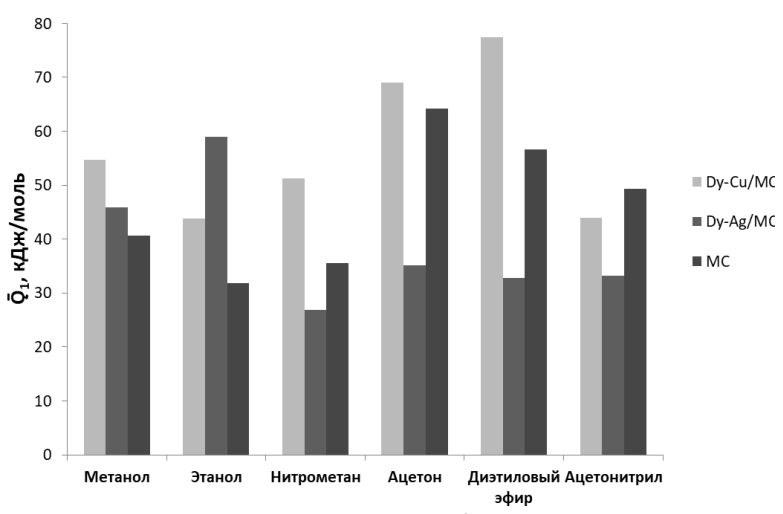

Рис. 7. Влияние модификатора на величины теплот ( $\left.\overline{\mathrm{Q}}_{1}\right)$ адсорбции полярных соединений на адсорбентах $\mathrm{MC}$, $\mathrm{Dy}-\mathrm{Ag} / \mathrm{MC}$ и $\mathrm{Dy}-\mathrm{Cu} / \mathrm{MC}$

Fig. 7. Influence of the modifier on the heat of adsorption (Q̄1) of polar compounds on MS adsorbents, Dy-Ag/MS and $\mathrm{Dy}-\mathrm{Cu} / \mathrm{MS}$

Установлено, что модифицирование приводит к сильным изменениям теплот адсорбции для соединений, склонных к различным типам специфических взаимодействий. На образцах $\mathrm{Dy}-\mathrm{Ag} / \mathrm{MC}$ и $\mathrm{Dy}-\mathrm{Cu} / \mathrm{MC}$ теплоты для спиртов выше, чем на немодифицированном образце МС. Наблюдается инверсия теплот адсорбции этанола и метанола на $\mathrm{Dy}-\mathrm{Cu} / \mathrm{MC}$ - адсорбция метанола характеризуется более высокой теплотой по сравнению с этанолом. По-видимому, это связано с влиянием пористости поверхности адсорбентов, так образец $\mathrm{Dy}-\mathrm{Cu} / \mathrm{MC}$ характеризуется меньшим объемом пор, по сравнению с образцом Dy-Ag/MC; поэтому молекуле метанола легче проникать в поры, чем молекулам этанола, и энергетическая составляющая адсорбции будет увеличиваться.

Теплоты адсорбции для нитрометана, ацетона, диэтилового эфира и ацетонитрила на образце Dy-Cu/MC выше, чем на образце Dy-Ag/MC. Можно предположить, что модифицирование медью, допированного диспрозием мезопористого силикагеля, увеличивает склонность этого адсорбента к диполь-дипольным и донорноакцепторным взаимодействиям. Разница в теплотах лежит в пределах от 10 кДж/моль для ацетонитрила и до 45 кДж/моль для диэтилового эфира. Установлено, что и поверхность немодифицированного МС проявляет большую склонность к диполь-дипольным и донорно-акцепторным взаимодействиям, чем образец Dу$\mathrm{Ag} / \mathrm{MC}$.

Для полного сравнительного анализа свойств адсорбентов важно иметь представление о превалирующей роли термодинамических характеристик процесса. Для этого проведен анализ зависимостей между теплотой и энтропией адсорбции, на основании которых можно оценить, какой термодинамический фактор является определяющим. На рис.8 приведены зависимости между теплотой и энтропией адсорбции для тестовых соединений на исследуемых адсорбентах.

Из рис. 8 следует, что определяющую роль при адсорбции тестовых соединений на синтезированных МС играет энтропийный фактор. Термодинамическая компенсационная зависимость проявляется в более явном виде для Dy-Ag/MC. 


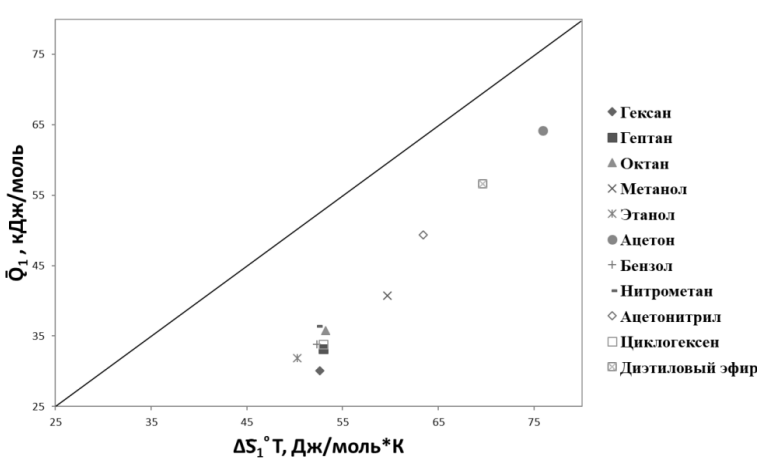

a

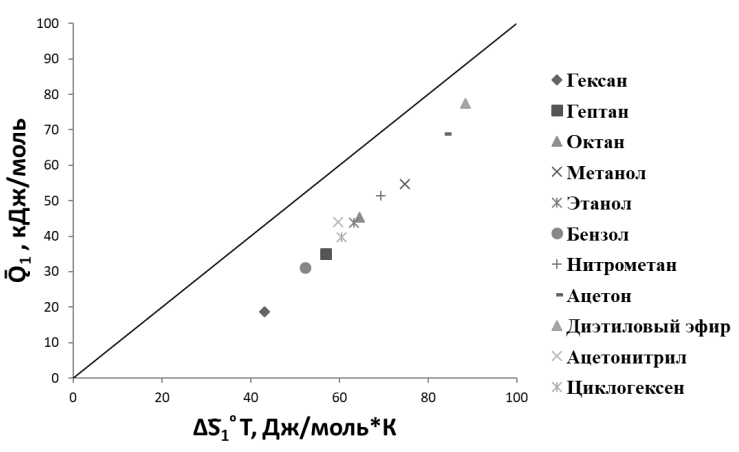

6

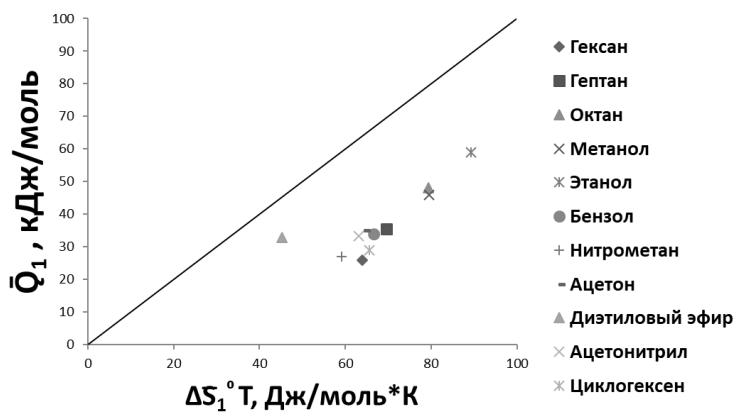

B

Рис. 8. Зависимость между теплой и энтропией адсорбции для тестовых адсорбатов: а) $\mathrm{MC}$, б) $\mathrm{Dy}-\mathrm{Cu} / \mathrm{MC}$, в) Dy-Ag/MC.

Fig. 8. The relationship between the heat and adsorption entropy for tested adsorbates: a) MS, b) Dy-Cu/MS, c) Dy-Ag/MS.

На модифицированных образцах возможны некоторые специфические взаимодействия между адсорбентом и адсорбатом, обусловленные электронным строением атомов металлов.

\section{Заключение}

Модифицирование металлами структуры мезопористого силикагеля приводит к уменьшению площади поверхности адсорбента, что, вероятно, связано с преимущественной локализацией металлов в порах мезопористой матрицы, а также позволяет в широком диапазоне варьировать средние объемы и диаметры пор.

Сравнение адсорбционных свойств MC, Dy-Cu/MC и Dy-Ag/MC показало, что $\mathrm{Dy}-\mathrm{Cu} / \mathrm{MC}$ проявляет наиболее ярко выраженные адсорбционные свойства по отношению к исследованным полярным тестовым органическим соединениям. Нa Dy$\mathrm{Cu} / \mathrm{MC}$ метанол адсорбируется с большей теплотой, чем этанол, что, вероятно, обусловливается низким значением объема пор образца.

Работа выполнена в рамках госзадания № FSSS-2020-0016 и РФФИ гранта № 19-33-90207.

\section{Список литературы}

1. Ahn Y., Kwak S.-Y. // Microporous and Mesoporous Materials. 2020. Vol. 306. P. 110410.
2. Gupta R., Gupta S.K., Pathak D.D. // Microporous and Mesoporous Materials. 2019. Vol. 288. P. 109577. 
3. Hachemaoui, M., Boukoussa, B., Mokhtar, A., Mekki, A. et al. // Materials Chemistry and Physics. 2020. Vol. 256. P. 123704.

4. Tan P., Jiang Y., Qi S.-C., Gao X.-J. et al. // Engineering. 2020. Vol. 6. pp. 569-576.

5. Федорова И.А., Шаповалова Е.Н., Староверов С.М., Шпигун О.А. // Сорбичионые и хроматографические проиессы. 2015. Т. 15. №. 5. C. 769-775

6. Al-Fatesh A.S., Hanan atia, Ibrahim A.A., Fakeeha A.H. et al. // Renewable Energy. 2019. Vol. 140. p. 658.

7. Zhu J., Peng X., Yao L., Shen J. et al. // International Journal of Hydrogen Energy. 2011. Vol. 36. pp. 7094-7104.

8. Zuo S., Wang X., Yang P., Qi C. // Catalysis Communications. 2017. Vol. 94. pp. 52-55.

9. Zhan W., Lu G., Guo Y., Guo Y. at al. // Journal of rare earths. 2008. Vol. 26. pp. 59-65.

10.Han Y., Wen B, Zhu M, Dai B. // Journal of Rare Earths. 2018. Vol. 36. pp. 367-373.

11.Budi C.S., Deka J.R., Saikia D., Kao H.-M. et al. // Journal of Hazardous Materials. 2020. Vol. 384. P. 121270.

12.Dening T.J., Zemlyanov D., Taylor L.S. // Journal of Controlled Release. 2019. Vol. 309. pp. 186-199.
13.O'Connor A.J., Hokura A., Kisler J.M., Shimazu S. et al. // Separation and Purification Technology. 2006. Vol. 48. pp. 197-201.

14.Карпов С.И., Рёсснер Ф., Селеменев В.Ф., Гульбин С.С. и др. // Сорбиионные и хроматографические прочессы. 2013. Т. 13. №. 2. C. $125-140$.

15.Mai, Z., Chen, J., Hu, Y., Liu, F. et al. // Journal of Colloid and Interface Science. 2017. Vol. 508. pp. 184-195.

16.Sarker M., Shin S., Jhung S.H. // Journal of Industrial and Engineering Chemistry. 2019. Vol. 74. pp. 158-163.

17.Policicchio A., Conte G., Stelitano S., Bonaventura C.P. et al. // Colloids and Surfaces A: Physicochemical and Engineering Aspects. 2020. Vol. 601. P. 125040.

18.Filippova E.O, Shafigulin R.V, Tokranov A.A, Shmelev A.A. et al. // J Chin Chem Soc. 2020. Vol. 67. pp. 1167-1173.

19.Shafigulin R. V., Filippova E.O., Shmelev A.A., Bulanova A.V. // Catalysis Letters. 2019. Vol. 149. No 4. pp. 916-928.

20.Шмелев А.А., Филиппова Е.О., Шафигулин Р.В., Буланова А.В. // Сорбционные и хроматографические проиессы. 2018. Т. 18. № 6. C.836-844.

\title{
Comparison of the adsorption properties of dysprosium-doped mesoporous silicas, modified with copper and silver, by inverse gas chromatography
}

\author{
(C) 2020 Filippova E.O., Shafigulin R.V., Vinogradov K.Yu., Bulanova A.V.
}

\author{
Samara University, Samara
}

Template synthesis is a promising method for the preparation of mesoporous silica, allowing controlling the pore diameter and surface area of MSM due to its large surface area ( $\left.700-1500 \mathrm{~m}^{2} / \mathrm{g}\right)$. It is possible to vary the selectivity of absorption of substances due to the rigid structure of the inorganic matrix, the availability of pores for the penetration of bulk molecules of organic substances, as well as the possibility of modifying materials. The studies performed during recent years were devoted to the production of new mesoporous materials doped with rare earth elements, materials with special, poorly studied properties. Previously, the authors of the study investigated the adsorption properties of mesoporous silica gel doped with dysprosium and modified with nickel and established that the incorporation of dysprosium into the structure of the material changes the adsorption properties of silica.

The aim of this study was the comparison of the adsorption properties of synthesised mesoporous silica gels doped with dysprosium and modified with copper and silver.

Two samples of mesoporous silica were synthesised by the template method: mesoporous silica doped with dysprosium and modified with copper (Dy-Cu/MS) and mesoporous silica doped with dysprosium and modified with silver (Dy-Ag/MS).

The textural and morphological properties of the samples were investigated by low-temperature nitrogen adsorption-desorption, scanning electron microscopy, X-ray phase analysis, inductively coupled plasma mass spectrometry, and X-ray fluorescence analysis. It was found that the introduction of dysprosium into the structure of a silica mesoporous material leads to a decrease in its surface area from $600 \mathrm{~m}^{2} / \mathrm{g}$ up to $514^{2 /}$ 
g. The synthesised samples were characterised by an average pore diameter of 3.4-7.2 $\mathrm{nm}$. The synthesised Dy-Ag/MS sample is characterised by the highest specific pore volume of $2.3 \mathrm{~cm}^{3} / \mathrm{g}$.

The thermodynamic characteristics of adsorption (differential heat of adsorption and change in standard differential molar entropy during adsorption) for tested organic compounds were obtained by the method of inverse gas chromatography. It was found that doping and the subsequent modification lead to changes in the heats of adsorption for compounds prone to different types of specific interactions. Thus, based on the comparison of the adsorption properties of the synthesised samples, it can be concluded that Dy$\mathrm{Cu} / \mathrm{MS}$ exhibits the most expressed adsorption properties in relation to the studied polar test compounds. On the $\mathrm{Dy}-\mathrm{Cu} / \mathrm{MS}$ sample, methanol was adsorbed with a higher heat than ethanol, which was probably due to the low pore volume of the sample.

Keywords: mesoporous silica gels, dysprosium doping, modification with copper and silver, thermodynamics of adsorption, inverse gas chromatography

\section{References}

1. Ahn Y., Kwak S.-Y., Microporous and Mesoporous Materials, 2020, Vol. 306, P. 110410. DOI: $10.1016 / \mathrm{j}$. micromeso.2020. 110410

2. Gupta R., Gupta S.K., Pathak D.D., Microporous and Mesoporous Materials, 2019, Vol. 288, Article 109577. DOI: 10.1016/j.micromeso.2019.109577

3. Hachemaoui, M., Boukoussa, B., Mokhtar, A., Mekki, A. et al., Materials Chemistry and Physics, 2020, Vol. 256, P. 123704. DOI: 10.1016/j.matchemphys.2020.123704

4. Tan P., Jiang Y., Qi S.-C., Gao X.-J. et al., Engineering, 2020, Vol. 6, pp. 569-576. DOI: 10.1016/j.eng.2020.03.005

5. Fedorova I.A., Shapovalova E.N., Staroverov S.M., Shpigun O.A., Sorbtsionnye $i$ khromatograficheskie protsessy, 2015, Vol. 15, No 5, pp. 769-775

6. Al-Fatesh A.S., Hanan atia, Ibrahim A.A., Fakeeha A.H. et al., Renewable Energy, 2019, Vol. 140, p. $658 . \quad$ DOI: 10.1016/j.renene.2019.03.082

7. Zhu J., Peng X., Yao L., Shen J. et al., International Journal of Hydrogen Energy, 2011, Vol. 36, pp. 7094-7104. DOI: 10.1016/j.ijhydene.2011.02.133

8. Zuo S., Wang X., Yang P., Qi C., Catalysis Communications, 2017, Vol. 94, pp. 52-55. DOI: 10.1016/j.catcom.2017.02.017

9. Zhan W., Lu G., Guo Y., Guo Y., Wang Y., Journal of rare earths, 2008, Vol. 26, pp. 59-65. DOI: 10.1016/S1002-0721(08)60038-1

10.Han Y, Wen B, Zhu M, Dai B, Journal of Rare Earths, 2018, Vol. 36, pp. 367-373. DOI: 10.1016/j.jre.2017.07.016

11.Budi C.S., Deka J.R., Saikia D., Kao H.-M. et al., Journal of Hazardous Materials, 2020,
Vol. 384, Article 1212700. DOI: 10.1016/i.jhazmat.2019.121270

12.Dening T.J., Zemlyanov D., Taylor L.S., Journal of Controlled Release, 2019, Vol. 309, $\mathrm{pp}$. 186-199.

DOI: 10.1016/j.jconrel.2019.06.028

13.O'Connor A.J., Hokura A., Kisler J.M., Shimazu S. et al., Separation and Purification Technology, 2006, Vol. 48, pp. 197-201. DOI: 10.1016/j.seppur.2005.07.007

14.Karpov S.I., Ressner F., Selemenev V.F., Gul'bin S.S. et al., Sorbtsionnye $i$ khromatograficheskie protsessy, 2013, Vol. 13, No 2, pp. 125-140.

15.Mai, Z., Chen, J., Hu, Y., Liu, F. et al., Journal of Colloid and Interface Science, 2017, Vol. 508, pp. 184-195. DOI: 10.1016/j.jcis.2017.07.027

16. Sarker M., Shin S., Jhung S.H., Journal of Industrial and Engineering Chemistry, 2019, Vol. 74, pp. 158-163. DOI: 10.1016/j.jiec.2019.02.022

17.Policicchio A., Conte G., Stelitano S., Bonaventura C.P. et al., Colloids and Surfaces A: Physicochemical and Engineering Aspects, 2020, Vol. 601, Article 125040. DOI: 10.1016/j.colsurfa.2020.125040

18. Filippova E.O,Shafigulin R.V, Tokranov A.A, Shmelev A.A., et al., J Chin Chem Soc., 2020, Vol. 67, pp. 1167-1173. DOI: $10.1002 /$ jccs. 201900440

19. Shafigulin R. V., Filippova E.O., Shmelev A.A., Bulanova A.V., Catalysis Letters, 2019, Vol. 149, No 4, pp. 916-928. DOI: 10.1007/s10562-019-02678-x

20.Shmelev A.A., Filippova E.O., Shafigulin R.V., Bulanova A.V., Sorbtsionnye i khromatograficheskie protsessy, 2018, Vol. 18, No 6, pp.836-844. 
Филиппова Елена Олеговна - аспирант кафедры физической химии и хроматографии, Самарский национальный исследовательский университет им. академика С.П.Королева, Самара

Виноградов Кирилл Юрьевич - аспирант кафедры физической химии и хроматографии, Самарский национальный исследовательский университет им. академика С.П.Королева, Самара

Шафигулин Роман Владимирович - доцент кафедры физической химии и хроматографии, к.х.н., Самарский национальный исследовательский университет им. академика С.П.Королева, Самара

Буланова Анджела Владимировна - профессор кафедры физической химии и хроматографии, д.х.н., Самарский национальный исследовательский университет им. академика С.П.Королева, Самара
Filippova Elena O. - the postgraduate student, department of physical chemistry and chromatography, Samara University, Samara. E-mail: fileona@mail.ru

Vinogradov Kirill Yu. - the postgraduate student, department of physical chemistry and chromatography, Samara University, Samara.

Shafigulin Roman V. - Ph.D. (chemistry), associate prof., department of physical chemistry and chromatography, Samara University, Samara. E-mail: shafiro@mail.ru

Bulanova Andzhela V. - prof., grand Ph.D (chemistry), department of physical chemistry and chromatography, Samara University, Samara. Email: av.bul@yandex.ru 